Supporting information

\title{
Kinetic Analysis of Electrochemical Oxygen Reduction over a Fe/N/C Catalyst Considering the Chemical Decomposition of $\mathrm{H}_{2} \mathrm{O}_{2}$
}

Yun Wu, ${ }^{a}$ Azhagumuthu Muthukrishnan, ${ }^{a, b}$ Shinsuke Nagata, ${ }^{a}$ and Yuta Nabae ${ }^{a, *}$

${ }^{a}$ Department of Materials Science and Engineering, Tokyo Institute of Technology, 2-12-1 S8-26, Ookayama, Meguro-ku, Tokyo 152-8522, Japan.

${ }^{b}$ School of Chemistry, Indian Institute of Science Education and Research Thiruvananthapuram, Maruthamala (P.O), Vithura, Thiruvananthapuram, Kerala, India. 
(a) $\mathrm{H}_{2} \mathrm{O}_{2}$ voltammogram

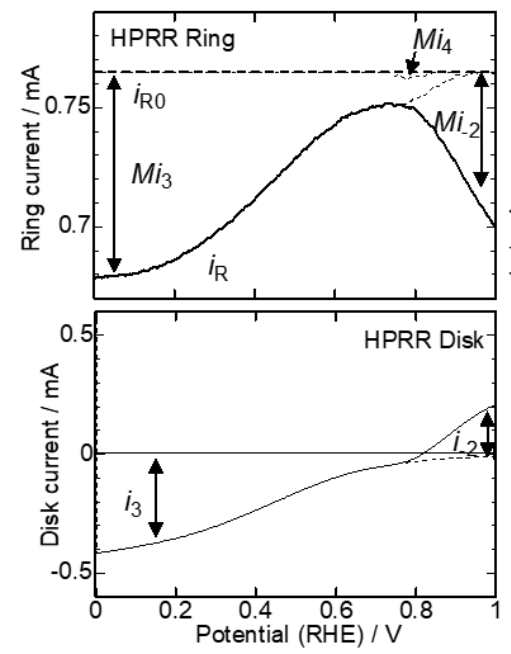

(d) ORR voltammogram

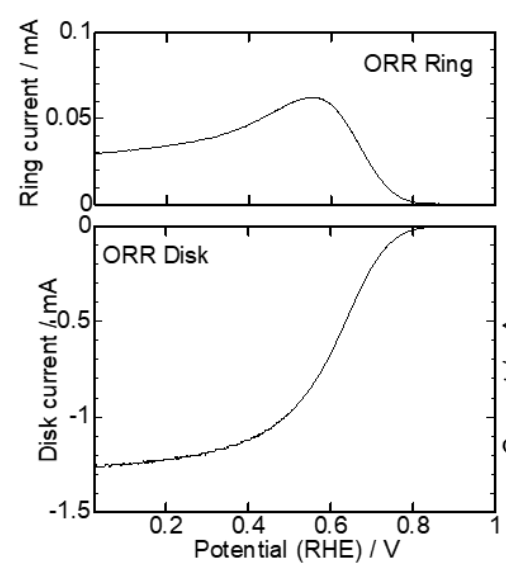

(b) $i_{-2}$ vs $i_{\mathrm{R}}$ plot

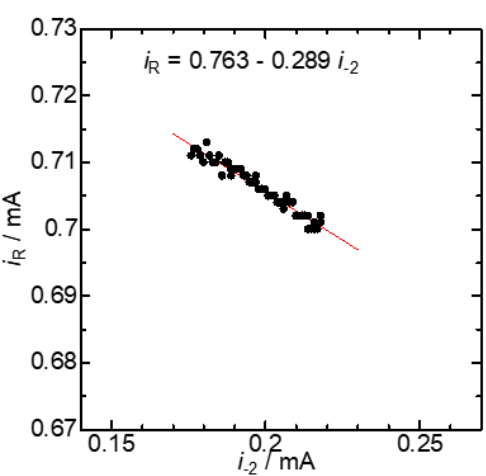

(e) ORR contribution$$
\text { 西 }
$$

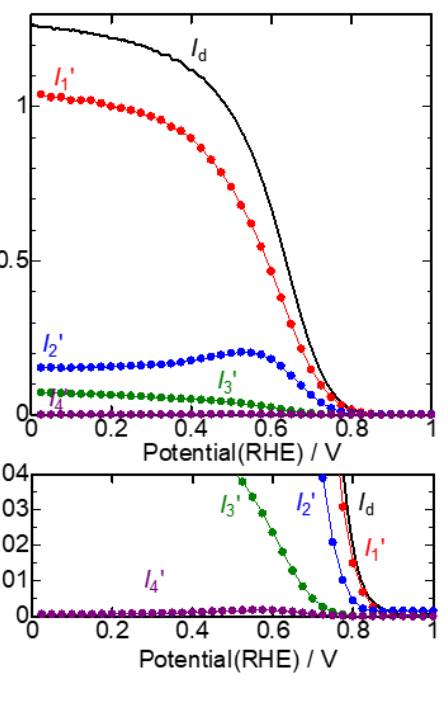

(c) $k$ for $\mathrm{H}_{2} \mathrm{O}_{2}$

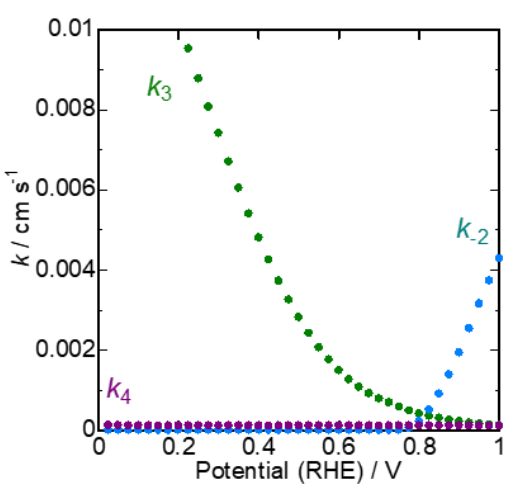

(f) $k^{\prime}$ for $\mathrm{O}_{2}$

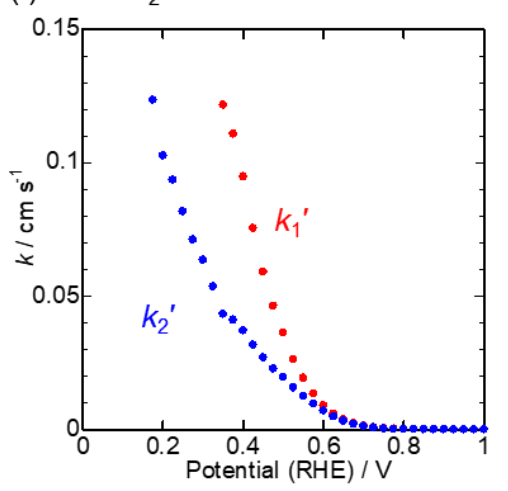

Figure. S1. (a) $\mathrm{H}_{2} \mathrm{O}_{2}$ voltammogram, (b) $i_{-2}$ vs. $i_{\mathrm{R}}$ plot at $0.95-1.0 \mathrm{~V}$, (c) kinetic constants for $\mathrm{H}_{2} \mathrm{O}_{2}$, (d) ORR voltammogram, (e) individual currents for ORR, and (f) kinetic constants for $\mathrm{O}_{2}$ before the loading density correction for a Fe/N/C catalyst. Catalyst loading: $80 \mu \mathrm{g} \mathrm{cm}^{-2}$. Rotation: $1600 \mathrm{rpm}$. Electrolyte: $0.5 \mathrm{M} \mathrm{H}_{2} \mathrm{SO}_{4}$ at room temperature. 
(a) $\mathrm{H}_{2} \mathrm{O}_{2}$ voltammogram

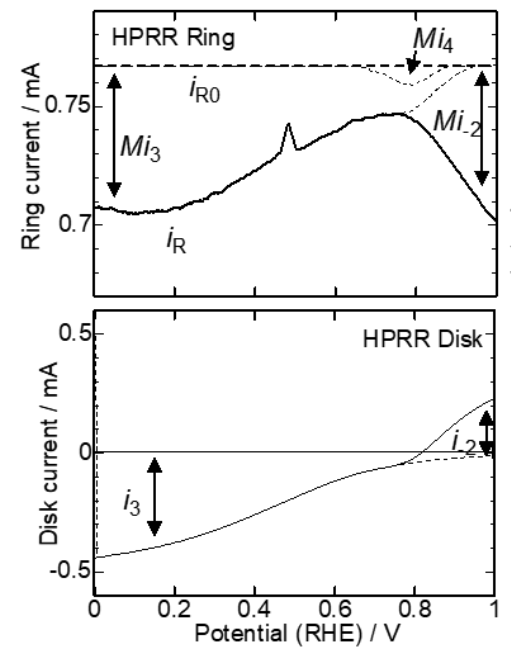

(d) ORR voltammogram

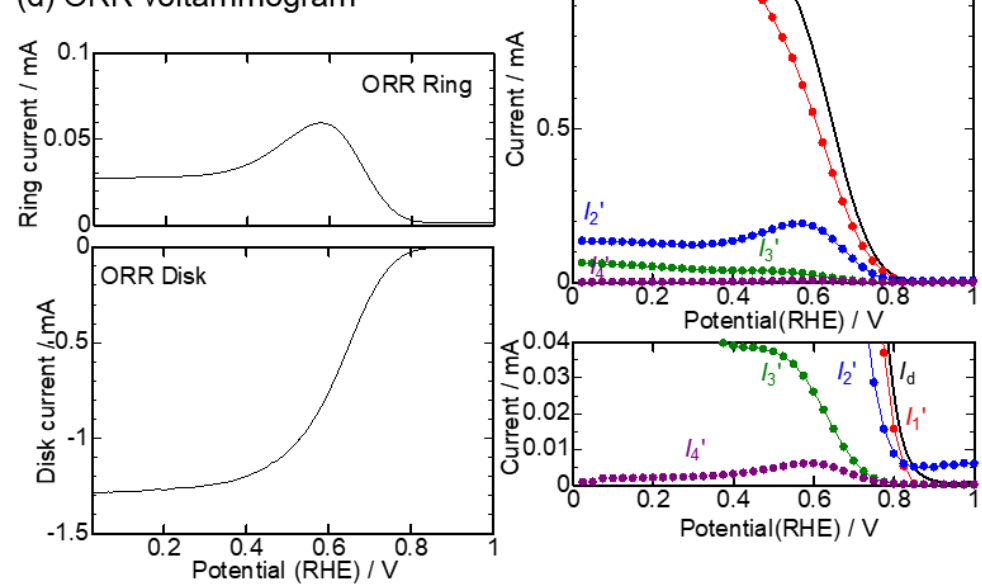

(c) $k$ for $\mathrm{H}_{2} \mathrm{O}_{2}$

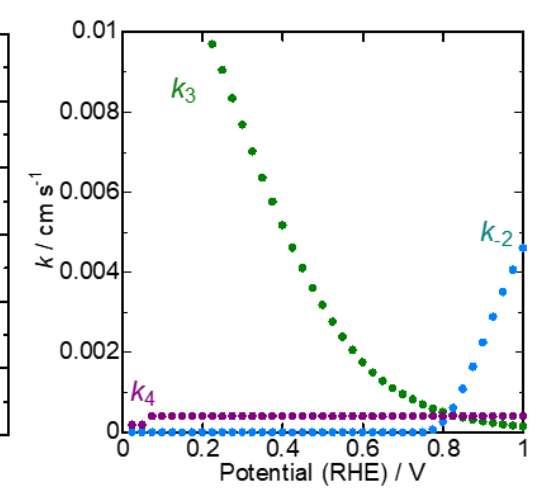

(e) ORR contribution$$
\text { (e) }
$$
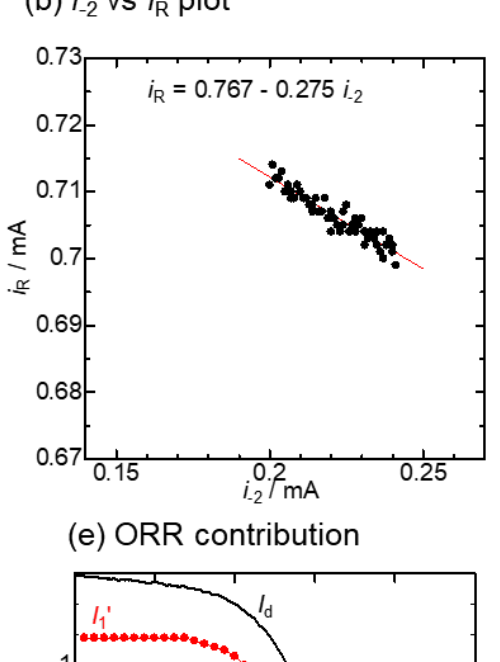

(f) $k^{\prime}$ for $\mathrm{O}_{2}$

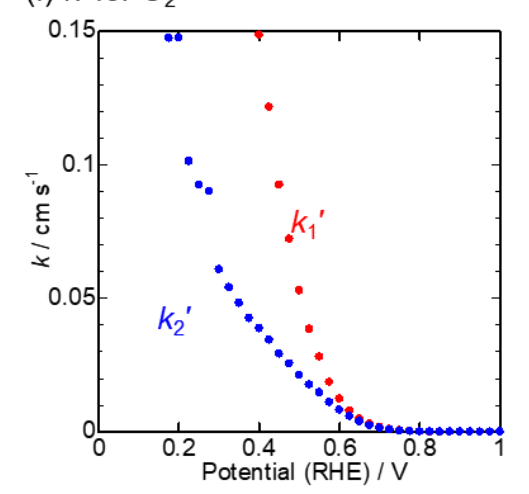

Figure. S2. (a) $\mathrm{H}_{2} \mathrm{O}_{2}$ voltammogram, (b) $i_{-2}$ vs. $i_{\mathrm{R}}$ plot at $0.95-1.0 \mathrm{~V}$, (c) kinetic constants for $\mathrm{H}_{2} \mathrm{O}_{2}$, (d) ORR voltammogram, (e) individual currents for ORR, and (f) kinetic constants for $\mathrm{O}_{2}$ before the loading density correction for a Fe/ $/ \mathrm{C}$ catalyst. Catalyst loading: $100 \mu \mathrm{g} \mathrm{cm}^{-2}$. Rotation: $1600 \mathrm{rpm}$. Electrolyte: $0.5 \mathrm{M} \mathrm{H}_{2} \mathrm{SO}_{4}$ at room temperature. 
(a) $\mathrm{H}_{2} \mathrm{O}_{2}$ voltammogram

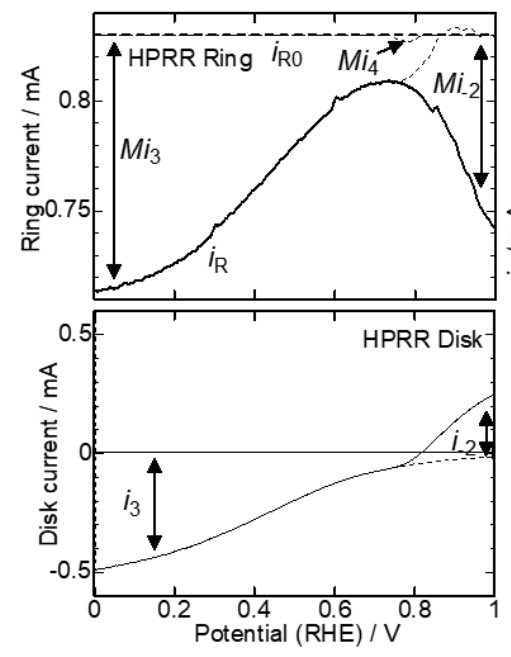

(d) ORR voltammogram
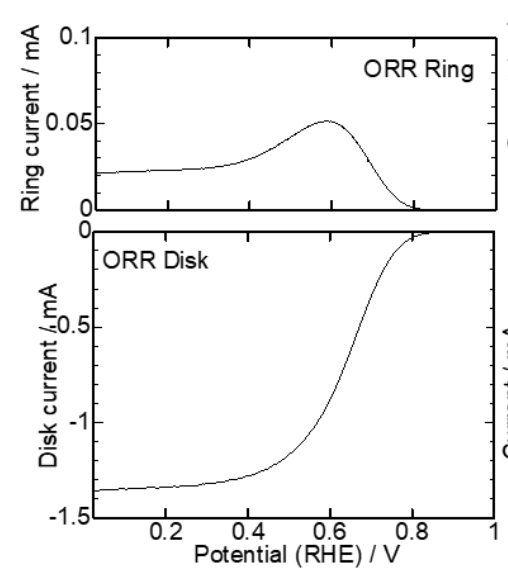

(b) $i_{-2}$ vs $i_{\mathrm{R}}$ plot

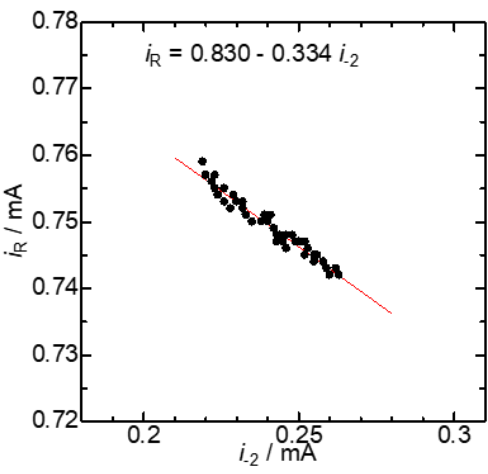

(e) ORR contribution

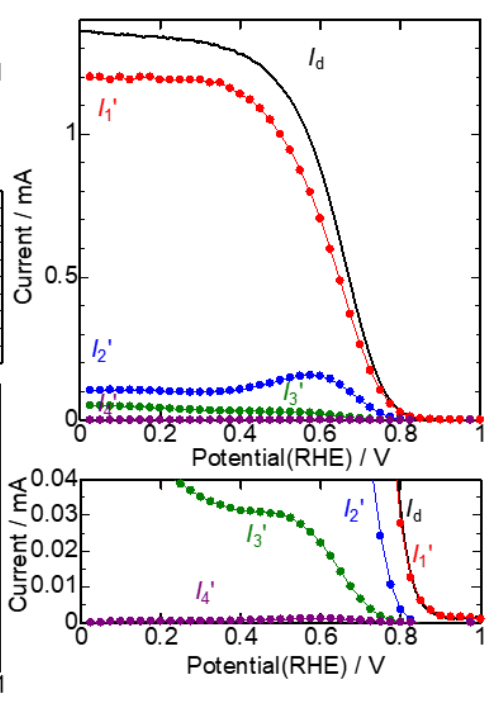

(c) $k$ for $\mathrm{H}_{2} \mathrm{O}_{2}$

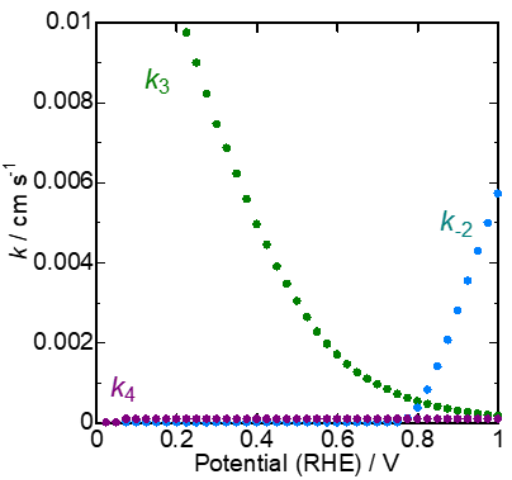

(f) $k^{\prime}$ for $\mathrm{O}_{2}$

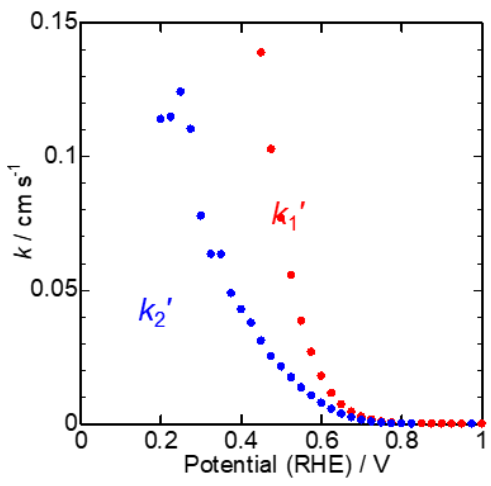

Figure. S3. (a) $\mathrm{H}_{2} \mathrm{O}_{2}$ voltammogram, (b) $i_{-2}$ vs. $i_{\mathrm{R}}$ plot at $0.95-1.0 \mathrm{~V}$, (c) kinetic constants for $\mathrm{H}_{2} \mathrm{O}_{2}$, (d) ORR voltammogram, (e) individual currents for ORR, and (f) kinetic constants for $\mathrm{O}_{2}$ before the loading density correction for a Fe/N/C catalyst. Catalyst loading: $120 \mu \mathrm{g} \mathrm{cm}^{-2}$. Rotation: $1600 \mathrm{rpm}$. Electrolyte: $0.5 \mathrm{M} \mathrm{H}_{2} \mathrm{SO}_{4}$ at room temperature. 